Research

\title{
Effects of inducible nitric oxide synthase inhibition or norepinephrine on the neurovascular coupling in an endotoxic rat shock model
}

\author{
Bernhard Rosengarten ${ }^{1}$, Stephanie Wolff ${ }^{1}$, Sabine Klatt ${ }^{1}$ and Ralf T Schermuly ${ }^{2}$
}

1Department of Neurology, Justus Liebig University Giessen, Am Steg 14, 35392 Giessen, Germany
2Department of Internal Medicine II, Justus Liebig University Giessen, Klinikstrasse 36, 35392 Giessen, Germany

Corresponding author: Bernhard Rosengarten, bernhard.rosengarten@neuro.med.uni-giessen.de

Received: 26 Jun 2009 Revisions requested: 22 Jul 2009 Revisions received: 23 Jul 2009 Accepted: 26 Aug 2009 Published: 26 Aug 2009

Critical Care 2009, 13:R139 (doi:10.1186/cc8020)

This article is online at: http://ccforum.com/content/13/4/R139

(C) 2009 Rosengarten et al.; licensee BioMed Central Ltd.

This is an open access article distributed under the terms of the Creative Commons Attribution License (http://creativecommons.org/licenses/by/2.0), which permits unrestricted use, distribution, and reproduction in any medium, provided the original work is properly cited.

\begin{abstract}
Introduction The inducible nitric oxide synthase (iNOS) plays a crucial role in early sepsis-related microcirculatory dysfunction. Compared to a catecholamine therapy we tested effects of a specific iNOS-inhibitor $(1400 \mathrm{~W})$ on the microcirculatory function in the brain.

Methods Seventy SD-rats (280-310 g) were divided into 1
control and 6 sepsis groups. Sepsis groups received 1 or $5 \mathrm{mg} /$
kg lipopolysaccharide (LPS) intravenously to induce a moderate
or severe sepsis syndrome. Thirty minutes later rats were further
randomized into subgroups receiving moderate volume therapy
alone or additionally continuous norepinephrine (NE) or $1400 \mathrm{~W}$
infusion. Separately, effects of $1400 \mathrm{~W}$ on neurofunctional
parameters were investigated in 3 rats without sepsis induction.
Performing electric forepaw-stimulation evoked potentials (N2-
P1 amplitude, P1-latency) and local hemodynamic responses
were recorded with surface electrodes and laser Doppler over
the somatosensory cortex at baseline and repeatedly after LPS
\end{abstract} administration. Cytokine levels (tumor necrosis factor-alpha (TNF $\alpha$ ), interleukin-6 (IL6), interferon-gamma (IFN $\gamma$ )) and cell destruction markers (neuron-specific enolase (NSE), S-100 calcium binding protein $\mathrm{B}(\mathrm{S} 100 \mathrm{~B}))$ were obtained at the end of experiments.

Results During sepsis progression resting cerebral blood flow increased and functionally activated hemodynamic responses decreased in a dose-dependent manner. Whereas $1400 \mathrm{~W}$ and NE improved blood pressure, only $1400 \mathrm{~W}$ stabilized resting flow levels. However, both regimens were ineffective on the functionally coupled flow responses and destruction markers were similar between groups.

Conclusions NE and 1400W appeared to be ineffective in mitigating the effects of sepsis on the neurovascular coupling. Other regimens are needed to protect the cerebral microcirculation under septic conditions.

\section{Introduction}

Sepsis and systemic inflammatory response syndromes are the leading causes of mortality in intensive care units $[1,2]$. Overt nitric oxide (NO) production by the inducible form of NO-synthases (iNOS) is assumed to play an important role in early sepsis-related vasoregulative failure $[3,4]$. In response to inflammatory stimuli $\mathrm{NO}$ levels increase rapidly within minutes to hours $[3,4]$ leading to hypotension [5-7] and refractoriness to vasopressor catecholamines [8]. Animals treated with selective iNOS-inhibitors or transgenic mice deficient in iNOS showed less hypotension and increased microvascular reactivity under septic conditions [9-11].

Regarding the cerebral circulation NO is intimately involved in the adequate blood flow distribution under physiologic conditions [12-14]. The excessive 100- to 1000 -fold increase in NO levels overrides the physiologic signals leading to a dissociation of the cerebral circulation. Although the overall perfusion is increased (cerebral hyperemia) $[7,15,16]$ it comes to a dysregulation on the microcirculative level $[16,17]$. As the brain is very dependent on an appropriate blood supply the microcir-

1400W: N-(3-(aminomethyl)benzyl)acetamidine; ELISA: enzyme-linked immunosorbent assay; IFN: interferon; IL: interleukin; iNOS: inducible nitric oxide synthase; LDF: laser-Doppler flowmetry; LPS: lipopolysaccharide; $\mathrm{NaCl}$ : sodium chloride; NE: norepinephrine; NO: nitric oxide; NSE: neuron specific enolase; SEP: somatosensory evoked potentials; TNF: tumor necrosis factor. 
culatory failure was in part suggested to best explain the early occurrence of sepsis-associated delirium $[17,18]$.

Whereas catecholamines can restore the macrocirculation there is growing evidence that they do not prevent the occurrence of microcirculatory dysfunction [19] Therefore, inhibition of the iNOS might be an interesting therapeutic regimen in sepsis syndromes. In this study, we compared protective effects of a specific iNOS-inhibitor N-(3-(aminomethyl)benzyl)acetamidine (1400W) with those of norepinephrine (NE) on the cerebral microcirculation as evaluated by the neurovascular coupling mechanism. To make comparison between a moderate or severe sepsis syndrome $1 \mathrm{mg} / \mathrm{kg}$ or $5 \mathrm{mg} / \mathrm{kg}$ lipopolysaccharide doses were given.

\section{Materials and methods General preparation}

All procedures performed on the animals were in strict accordance with the National Institutes of Health Guide for Care and Use of Laboratory Animals and approved by the local Animal Care and Use Committee.

Adult male SD-rats (weighing 280 to $310 \mathrm{~g}$ ) were initially anesthetized with 1.5 to $3 \%$ isoflurane in a $7: 3$ nitrous oxide $\left(\mathrm{N}_{2} \mathrm{O}\right)$ /oxygen mixture of gases, tracheotomized, paralyzed with pancuronium bromide $(0.2 \mathrm{mg} / \mathrm{kg} / \mathrm{h})$, and artificially ventilated (Harvard Rodent Ventilator; Harvard, South Natick, MA, USA). Arterial blood gas analyses and $\mathrm{pH}$ were measured repeatedly as needed and at least every 30 minutes (Blood gas analyzer model Rapidlab 348, Bayer Vital GmbH, Fernwald, Germany). Also, glucose and lactate levels were measured repeatedly (Glukometer Elite XL, Bayer Vital GmbH, Fernwald, Germany; Lactate pro, Arkray Inc. European Office, Düsseldorf, Germany). Glucose was kept in the physiologic range by injections of $0.5 \mathrm{ml} 20 \%$ glucose as needed. The right femoral artery and vein were cannulated for blood pressure recording, blood sampling, and drug administration. Rectal body temperature was maintained at $37^{\circ} \mathrm{C}$ using a feedback-controlled heating pad.

The head of the animals was fixed in a stereotaxic frame, the apex of the skull was exposed, and the bone over the left parietal cortex was thinned with a saline-cooled drill to allow transcranial laser-Doppler flowmetry (LDF) [20]. The laser probe (BRL-100, Harvard Apparatus, Holliston, MA, USA) was placed $3.5 \mathrm{~mm}$ lateral and $1 \mathrm{~mm}$ rostral to the bregma in accordance with the coordinates of the somatosensory cortex; this location corresponds closely to the region of maximal hemodynamic response during contralateral forepaw stimulation [21-23]. The laser-Doppler signal and the systemic mean arterial blood pressure were recorded continuously and processed on a personal computer running a data acquisition software (Neurodyn, HSE, March-Hugstetten, Germany). As the laser Doppler measures flow changes rather than absolute values, resting LDF signals are given in arbitrary units. However, evoked signal changes can be used to assess flow changes and are given in percent-changes from baseline [21,22].

Somatosensory stimulation was carried out with electrical pulses applied by small needle electrodes inserted under the skin of the right forepaw (PSM Module 676, HSE, March-Hugstetten, Germany). Electric brain activity was recorded monopolarily with an active calomel electrode at $0.5 \mathrm{~mm}$ behind the laser probe and an indifferent calomel electrode placed on the nasal bone. Signals were recorded and amplified (BPA Module 675, HSE, March-Hugstetten, Germany) and somatosensory evoked potentials (SEP) were averaged using the Neurodyn acquisition software (HSE, March-Hugstetten, Germany). Evoked potential amplitudes were calculated from the N2-P1-amplitude differences and the latency between the start of stimulation and occurrence of the P1-peak was obtained.

Approximately 60 minutes before the stimulation experiments, isoflurane $/ \mathrm{N}_{2} \mathrm{O}$ anesthesia was discontinued and replaced by intravenous application of $\alpha$-chloralose $(80 \mathrm{mg} / \mathrm{kg}$; SigmaAldrich Chemie $\mathrm{GmbH}$, Taufkirchen, Germany). Supplementary doses of chloralose $(30 \mathrm{mg} / \mathrm{kg}$ ) were given every hour. During chloralose anesthesia, the animals were ventilated with a nitrogen/oxygen mixture of $1 / 1$.

\section{Neurovascular coupling measurement}

Somatosensory activation was carried out by electrical stimulation of the right forepaw with rectangular pulses of $0.3 \mathrm{~ms}$ width and a repetition frequency of $2 \mathrm{~Hz}$ for 30 seconds. The stimulation current was kept constant at $1.5 \mathrm{~mA}$ so that systemic blood pressure changes did not occur [21-23]. Allowing a rest of 30 seconds after each stimulation train, activationrest cycles were repeated 10 times to increase signal to noise ratio. Flow velocity responses were averaged and relative responses were calculated in relation to the resting phase, setting the resting phase to zero. The evoked flow velocity responses were calculated from the averaged relative flow velocity signals under conditions of stimulation.

\section{Clinical chemistry}

At the end of the experiments blood samples were drawn into tubes containing aprotinin (Trasylol, Bayer AG, Leverkusen, Germany), immediately centrifugated and separated, after which plasma was stored at $-80^{\circ} \mathrm{C}$ until analyses. The neuronspecific enolase (NSE) levels were determined using an ELISA (NSE EIA kit; Hoffmann-La Roche, Basel, Switzerland). The S-100B protein was determined with an immunoluminometric assay (Sangtec 100 LIA; Sangtec Medical, Bromma, Sweden) using monoclonal antibodies specific for the beta subunit of the S-100 protein. Cytokine analysis were performed for IL-6, TNF $\alpha$, interferon (IFN) $\gamma$ using commercialized rat ELISA sets (BD Bioscience, Heidelberg, Germany). 


\section{Study design}

Each 10 rats were subjected to one of the following groups: control, $1 \mathrm{mg} / \mathrm{kg}$ LPS (LPS Escherichia coli, O111:B4, Sigma-Aldrich Chemie GmbH, Germany), 5 mg/kg LPS, 1 mg/ $\mathrm{kg} \mathrm{LPS} \mathrm{+} \mathrm{1400W,} 1 \mathrm{mg} / \mathrm{kg}$ LPS + NE, $5 \mathrm{mg} / \mathrm{kg} \mathrm{LPS} \mathrm{+}$ $1400 \mathrm{~W}, 5 \mathrm{mg} / \mathrm{kg}$ LPS + NE. LPS was dissolved in $0.5 \mathrm{ml}$ $0.9 \%$ sodium chloride $(\mathrm{NaCl})$. LPS was given within two to three minutes. The control group received $0.5 \mathrm{ml}$ vehicle. A moderate volume therapy of 1 to $6 \mathrm{ml} / \mathrm{kg} / \mathrm{h} 0.9 \% \mathrm{NaCl}$ was allowed in all groups. Thirty minutes after sepsis induction $1400 \mathrm{~W}$ was given as a bolus of $7.5 \mathrm{mg} / \mathrm{kg}$ followed by a continuous infusion at a rate of $7.5 \mathrm{mg} / \mathrm{kg} / \mathrm{h}$. NE was given in doses between 0.01 and $10 \mu \mathrm{g} / \mathrm{kg} / \mathrm{min}$ to stabilize mean blood pressure in the lower physiologic range between 90 and $100 \mathrm{mmHg}$.

Prior to and then after LPS administration SEPs, evoked and resting cerebral blood flow velocity levels and blood pressure were measured up to 270 minutes.

In an additional group $(n=3)$, we investigated the effects of the same dose of $1400 \mathrm{~W}$ without sepsis induction in healthy rats.

\section{Statistics}

If appropriate, a two-way analysis of variance was performed to assess differences within and between groups. In case of significance a Fischer post-hoc test was applied. If assumptions of normal distribution and equality of variances could not be assured, a nonparametric Friedman test was undertaken instead (Statview, SAS, Cary, NA, USA). The significance level was set to $P<0.05$.

\section{Results}

No rat died from LPS injection. Table 1 shows the group averaged data for partial pressure of carbon dioxide, $\mathrm{pH}$, glucose, lactate, and hemoglobin content. Partial pressure of oxygen levels remained in the range of 240 to $250 \mathrm{mmHg}$ in all groups throughout experiments and therefore were not specified in the table. Table 2 indicates the group data for blood pressure together with the resting LDF signal, N2-P1 potential amplitude, P1 latency, and evoked flow velocity response. The cytokines as well as the cell destruction markers are given in Table 3.

In non-septic rats, $1400 \mathrm{~W}$ did not result in changes in the following data: blood pressure (121 $\pm 11 \mathrm{vs} .125 \pm 6 \mathrm{mmHg}$; not significant), glucose levels ( $60 \pm 9 \mathrm{vs} .57 \pm 6 \mathrm{mmol} / \mathrm{L}$; not significant), resting cerebral blood flow (135 \pm 25 vs. $142 \pm 28$; not significant), evoked flow responses ( $20 \pm 7$ vs. $20 \pm 8 \%$; not significant), SEP amplitudes (16 \pm 6 vs. $15 \pm 4 \mu \mathrm{V}$; not significant), or $\mathrm{P} 1$-latencies (10 \pm 2 vs. $10 \pm 1 \mathrm{~ms}$; not significant).

\section{General findings}

With LPS-administration, rats developed signs of a severe sepsis syndrome characterized by a considerable drop in blood pressure ( $1 \mathrm{mg} / \mathrm{kg}$ LPS: $63 \pm 10 \mathrm{mmHg} ; 5 \mathrm{mg} / \mathrm{kg}$ LPS: $56 \pm 11 \mathrm{mmHg}$ ), occurrence of metabolic acidosis $(1 \mathrm{mg} / \mathrm{kg}$ LPS: $7.48 \pm 0.04 ; 5 \mathrm{mg} / \mathrm{kg}$ LPS: $7.46 \pm 0.04)$, and an increase in the lactate levels ( $1 \mathrm{mg} / \mathrm{kg}$ LPS: $2.5 \pm 0.8 ; 5 \mathrm{mg} /$ kg LPS: $2.4 \pm 0.7 \mathrm{mmol} / \mathrm{l}$ ) as indicated in Table 1. Cytokine levels increased in all sepsis groups without differences between groups (Table 3). Resting flow levels were significantly induced in both LPS + NE groups by approximately $50 \%$, whereas 1400W remained neutral (Table 2). Compared with non-treated groups NE improved blood pressure levels effectively in both groups $(95 \pm 17 \mathrm{mmHg}$ and $92 \pm 15$

\section{Table 1}

\begin{tabular}{|c|c|c|c|c|c|c|c|c|c|c|}
\hline & \multicolumn{2}{|c|}{ Glucose (mg/dL) } & \multicolumn{2}{|c|}{ Lactate (mmol/L) } & \multicolumn{2}{|l|}{$\mathrm{pH}$} & \multicolumn{2}{|c|}{$\mathrm{pCO}_{2}(\mathrm{mmHg})$} & \multicolumn{2}{|c|}{ Hemoglobin (mg/l) } \\
\hline & Base & End & Base & End & Base & End & Base & End & Base & End \\
\hline Control & $78 \pm 12$ & $82 \pm 8$ & - & $0.7 \pm 0.7$ & $7.57 \pm 0.06$ & $7.53 \pm 0.05$ & $32.6 \pm 4.5$ & $33.5 \pm 2.2$ & $137 \pm 7$ & $135 \pm 6$ \\
\hline $1 \mathrm{mg} / \mathrm{kg}$ & $89 \pm 18$ & $55 \pm 9^{\star \star \star}$ & - & $2.5 \pm 0.8^{\star \star \star}$ & $7.53 \pm 0.05$ & $7.48 \pm 0.04^{*}$ & $34.5 \pm 5.1$ & $32.8 \pm 2.2$ & $137 \pm 6$ & $124 \pm 15^{\star}$ \\
\hline $5 \mathrm{mg} / \mathrm{kg}$ & $74 \pm 19$ & $46 \pm 15^{\star \star \star}$ & - & $2.4 \pm 0.7^{\star \star \star}$ & $7.51 \pm 0.05$ & $\begin{array}{l}7.46 \pm \\
0.04^{\star \star \star}\end{array}$ & $35.5 \pm 4.1$ & $32.3 \pm 1.7$ & $133 \pm 9$ & $122 \pm 15^{\star \star}$ \\
\hline$\underset{\mathrm{NE}}{1 \mathrm{mg} / \mathrm{kg} .+}$ & $85 \pm 15$ & $68 \pm 14^{*}$ & - & $1.4 \pm 0.4^{\star \star \star}$ & $7.52 \pm 0.05$ & $7.48 \pm 0.05^{\star}$ & $35.4 \pm 4.0$ & $34.3 \pm 3.1$ & $139 \pm 10$ & $119 \pm 19^{\star *}$ \\
\hline $5 \mathrm{mg} / \mathrm{kg}+\mathrm{NE}$ & $76 \pm 14$ & $70 \pm 23$ & - & $1.5 \pm 0.6^{\star \star \star}$ & $7.52 \pm 0.03$ & $7.46 \pm 0.05^{\star \star}$ & $34.8 \pm 3.2$ & $32.8 \pm 2.5$ & $145 \pm 12$ & $122 \pm 17^{\star \star}$ \\
\hline $\begin{array}{l}1 \mathrm{mg} / \mathrm{kg}+ \\
1400 \mathrm{~W}\end{array}$ & $78 \pm 14$ & $51 \pm 7^{\star \star \star}$ & - & $2.5 \pm 0.6^{\star \star \star}$ & $7.53 \pm 0.06$ & $7.46 \pm 0.09^{\star \star}$ & $36.4 \pm 4.8$ & $35.2 \pm 2.6$ & $142 \pm 12$ & $121 \pm 18^{\star \star}$ \\
\hline $\begin{array}{l}5 \mathrm{mg} / \mathrm{kg}+ \\
1400 \mathrm{~W}\end{array}$ & $73 \pm 15$ & $46 \pm 8^{\star \star \star}$ & - & $2.4 \pm 0.4^{\star \star \star}$ & $7.52 \pm 0.03$ & $\begin{array}{l}7.46 \pm \\
0.04^{\star \star \star}\end{array}$ & $34.8 \pm 3.4$ & $34.3 \pm 1.4$ & $139 \pm 9$ & $120 \pm 15^{\star \star}$ \\
\hline
\end{tabular}

Data are given as mean \pm standard deviation (SD) together with statistical results. Significance is given as: ${ }^{*} P<0.05 ;{ }^{* \star} P<0.01 ;{ }^{* \star \star} P<0.001$ compared with baseline. $1400 \mathrm{~W}=\mathrm{N}-\left(3\right.$-(aminomethyl) benzyl) acetamidine; $\mathrm{NE}=$ norepinephrine; $\mathrm{pCO}_{2}=$ partial pressure of carbon dioxide. 
Table 2

Group averaged data for mean blood pressure, somatosensory evoked potentials, P1 latencies, evoked flow velocity responses, and resting LDFV signal, for the different time points of the experiment

\begin{tabular}{|c|c|c|c|c|c|c|c|c|}
\hline & & Baseline & $30 \mathrm{~min}$ & $60 \min$ & $120 \mathrm{~min}$ & $180 \mathrm{~min}$ & $240 \mathrm{~min}$ & $270 \mathrm{~min}$ \\
\hline Mean BP $(\mathrm{mmHg})$ & Control & $108 \pm 12$ & $108 \pm 9$ & $101 \pm 12$ & $103 \pm 11$ & $104 \pm 14$ & $108 \pm 18$ & $107 \pm 14$ \\
\hline Mean BP $(\mathrm{mmHg})$ & $1 \mathrm{mg} / \mathrm{kg}$ & $110 \pm 11$ & $84 \pm 13^{\star \star *}$ & $65 \pm 8^{\star \star \star}$ & $66 \pm 10^{\star \star *}$ & $62 \pm 12^{\star \star \star}$ & $56 \pm 9^{\star \star \star}$ & $63 \pm 10^{* * *}$ \\
\hline Mean BP $(\mathrm{mmHg})$ & $5 \mathrm{mg} / \mathrm{kg}$ & $115 \pm 10$ & $83 \pm 17^{\star \star \star}$ & $62 \pm 7^{\star \star \star}$ & $61 \pm 12^{\star \star \star}$ & $54 \pm 14^{\star \star \star}$ & $50 \pm 7^{\star \star \star}$ & $56 \pm 11^{\star \star \star}$ \\
\hline Mean BP (mmHg) & $1 \mathrm{mg} / \mathrm{kg}+\mathrm{NE}$ & $108 \pm 12$ & $84 \pm 14^{\star \star \star}$ & $99 \pm 12$ & $92 \pm 11$ & $97 \pm 11$ & $94 \pm 15$ & $95 \pm 15$ \\
\hline Mean BP $(\mathrm{mmHg})$ & $5 \mathrm{mg} / \mathrm{kg}+\mathrm{NE}$ & $104 \pm 10$ & $79 \pm 13^{\star \star \star}$ & $93 \pm 19$ & $90 \pm 13$ & $94 \pm 12$ & $94 \pm 16$ & $92 \pm 13$ \\
\hline Mean BP $(\mathrm{mmHg})$ & $1 \mathrm{mg} / \mathrm{kg}+1400 \mathrm{~W}$ & $106 \pm 13$ & $79 \pm 18^{\star \star \star}$ & $80 \pm 7^{\star \star}$ & $83 \pm 10^{\star *}$ & $85 \pm 10^{\star \star}$ & $79 \pm 10^{\star \star \star}$ & $82 \pm 12^{\star \star \star}$ \\
\hline Mean BP $(\mathrm{mmHg})$ & $5 \mathrm{mg} / \mathrm{kg}+1400 \mathrm{~W}$ & $110 \pm 11$ & $73 \pm 16^{\star \star \star}$ & $77 \pm 11^{\star \star}$ & $81 \pm 8^{\star \star}$ & $82 \pm 10^{\star \star}$ & $78 \pm 13^{\star \star \star}$ & $76 \pm 12^{\star \star \star}$ \\
\hline SEP $(\mu \mathrm{V})$ & Control & $21 \pm 4$ & $20 \pm 5$ & $21 \pm 5$ & $21 \pm 5$ & $20 \pm 4$ & $20 \pm 4$ & $20 \pm 4$ \\
\hline $\operatorname{SEP}(\mu \mathrm{V})$ & $1 \mathrm{mg} / \mathrm{kg}$ & $21 \pm 7$ & $18 \pm 6$ & $15 \pm 5^{\star \star}$ & $15 \pm 4^{\star \star \star}$ & $12 \pm 3^{\star \star \star}$ & $13 \pm 3^{\star \star \star}$ & $12 \pm 4^{\star \star \star}$ \\
\hline SEP $(\mu \mathrm{V})$ & $5 \mathrm{mg} / \mathrm{kg}$ & $20 \pm 5$ & $18 \pm 5$ & $15 \pm 5^{\star \star}$ & $15 \pm 5^{\star \star \star}$ & $13 \pm 4^{\star \star \star}$ & $10 \pm 3^{\star \star \star}$ & $7 \pm 2^{\star \star \star}$ \\
\hline SEP $(\mu \mathrm{V})$ & $1 \mathrm{mg} / \mathrm{kg}+\mathrm{NE}$ & $21 \pm 4$ & $18 \pm 4$ & $18 \pm 3^{*}$ & $17 \pm 3^{\star \star}$ & $16 \pm 3^{\star \star}$ & $16 \pm 3^{\star \star}$ & $16 \pm 3^{\star \star}$ \\
\hline SEP $(\mu \mathrm{V})$ & $5 \mathrm{mg} / \mathrm{kg}+\mathrm{NE}$ & $21 \pm 7$ & $17 \pm 4$ & $15 \pm 4^{\star \star}$ & $15 \pm 4^{\star \star \star}$ & $13 \pm 2^{\star \star \star}$ & $12 \pm 2^{\star \star \star}$ & $14 \pm 3^{\star \star \star}$ \\
\hline $\operatorname{SEP}(\mu \mathrm{V})$ & $1 \mathrm{mg} / \mathrm{kg}+1400 \mathrm{~W}$ & $22 \pm 3$ & $18 \pm 3$ & $11 \pm 2^{\star \star \star \star}$ & $11 \pm 2^{\star \star \star}$ & $10 \pm 2^{\star \star \star \star}$ & $9 \pm 2^{\star \star \star}$ & $9 \pm 1^{\star \star \star}$ \\
\hline $\operatorname{SEP}(\mu \mathrm{V})$ & $5 \mathrm{mg} / \mathrm{kg}+1400 \mathrm{~W}$ & $21 \pm 3$ & $17 \pm 4$ & $11 \pm 2^{\star \star \star}$ & $11 \pm 2^{\star \star \star}$ & $10 \pm 1^{\star \star \star}$ & $10 \pm 2^{\star \star \star}$ & $10 \pm 2^{\star \star \star}$ \\
\hline P1 latency (ms) & Control & $11 \pm 1$ & $11 \pm 1$ & $11 \pm 2$ & $11 \pm 1$ & $12 \pm 1$ & $11 \pm 1$ & $12 \pm 1$ \\
\hline P1 latency (ms) & $1 \mathrm{mg} / \mathrm{kg}$ & $11 \pm 0.4$ & $11 \pm 1$ & $12 \pm 1^{*}$ & $12 \pm 1$ & $12 \pm 1$ & $12 \pm 2$ & $13 \pm 1$ \\
\hline P1 latency (ms) & $5 \mathrm{mg} / \mathrm{kg}$ & $11 \pm 1$ & $12 \pm 1$ & $12 \pm 1^{*}$ & $12 \pm 2$ & $12 \pm 1$ & $13 \pm 2$ & $13 \pm 2$ \\
\hline P1 latency (ms) & $1 \mathrm{mg} / \mathrm{kg}+\mathrm{NE}$ & $12 \pm 0.5$ & $12 \pm 1$ & $12 \pm 1$ & $12 \pm 1$ & $12 \pm 1$ & $12 \pm 1$ & $12 \pm 1$ \\
\hline P1 latency (ms) & $5 \mathrm{mg} / \mathrm{kg}+\mathrm{NE}$ & $11 \pm 1$ & $12 \pm 0.4$ & $12 \pm 0.5^{*}$ & $12 \pm 2$ & $12 \pm 1$ & $12 \pm 1$ & $14 \pm 3$ \\
\hline P1 latency (ms) & $1 \mathrm{mg} / \mathrm{kg}+1400 \mathrm{~W}$ & $12 \pm 0.5$ & $13 \pm 1^{\star \star}$ & $14 \pm 1^{\star \star \star}$ & $14 \pm 1^{\star \star \star}$ & $15 \pm 2^{\star \star \star}$ & $15 \pm 3^{\star \star \star}$ & $15 \pm 2^{\star \star}$ \\
\hline P1 latency (ms) & $5 \mathrm{mg} / \mathrm{kg}+1400 \mathrm{~W}$ & $12 \pm 0.5$ & $13 \pm 1^{\star *}$ & $14 \pm 1^{\star \star \star}$ & $14 \pm 1^{\star \star \star}$ & $14 \pm 1^{\star \star \star}$ & $14 \pm 1^{\star \star \star}$ & $15 \pm 1^{*}$ \\
\hline EFVR (\%) & Control & $20 \pm 8$ & $20 \pm 6$ & $20 \pm 5$ & $16 \pm 6$ & $17 \pm 5$ & $17 \pm 4$ & $18 \pm 4$ \\
\hline EFVR (\%) & $1 \mathrm{mg} / \mathrm{kg}$ & $22 \pm 10$ & $14 \pm 7$ & $10 \pm 4^{\star \star}$ & $10 \pm 4^{*}$ & $10 \pm 4^{\star \star}$ & $11 \pm 5^{\star \star}$ & $10 \pm 5^{\star \star \star}$ \\
\hline EFVR (\%) & $5 \mathrm{mg} / \mathrm{kg}$ & $24 \pm 7$ & $16 \pm 7$ & $7 \pm 2^{\star \star \star}$ & $6 \pm 2^{\star \star}$ & $5 \pm 2^{\star \star \star}$ & $6 \pm 2^{\star \star \star}$ & $4 \pm 2^{\star \star \star}$ \\
\hline EFVR (\%) & $1 \mathrm{mg} / \mathrm{kg}+\mathrm{NE}$ & $23 \pm 7$ & $18 \pm 6$ & $16 \pm 10$ & $14 \pm 10$ & $11 \pm 8^{\star \star}$ & $10 \pm 7^{\star \star \star}$ & $9 \pm 6^{\star \star \star}$ \\
\hline EFVR (\%) & $5 \mathrm{mg} / \mathrm{kg}+\mathrm{NE}$ & $23 \pm 7$ & $14 \pm 6$ & $10 \pm 7^{\star \star}$ & $5 \pm 3^{\star \star \star}$ & $3 \pm 3^{\star \star \star}$ & $4 \pm 3^{\star \star \star}$ & $5 \pm 4^{\star \star \star}$ \\
\hline EFVR (\%) & $1 \mathrm{mg} / \mathrm{kg}+1400 \mathrm{~W}$ & $18 \pm 5$ & $16 \pm 8$ & $8 \pm 4^{\star \star \star}$ & $7 \pm 3^{* *}$ & $6 \pm 2^{\star \star \star}$ & $5 \pm 4^{* * *}$ & $5 \pm 3^{* \star *}$ \\
\hline EFVR (\%) & $5 \mathrm{mg} / \mathrm{kg}+1400 \mathrm{~W}$ & $24 \pm 7$ & $20 \pm 10$ & $7 \pm 4^{\star \star \star}$ & $8 \pm 5^{\star \star}$ & $7 \pm 5^{\star \star \star}$ & $6 \pm 3^{\star \star \star}$ & $5 \pm 4^{\star \star \star}$ \\
\hline Resting LDF (U) & Control & $176 \pm 49$ & $166 \pm 38$ & $170 \pm 39$ & $178 \pm 33$ & $178 \pm 35$ & $183 \pm 32$ & $186 \pm 32$ \\
\hline Resting LDF (U) & $1 \mathrm{mg} / \mathrm{kg}$ & $167 \pm 60$ & $180 \pm 66$ & $185 \pm 70$ & $200 \pm 95$ & $201 \pm 94$ & $203 \pm 101$ & $213 \pm 110$ \\
\hline Resting LDF (U) & $5 \mathrm{mg} / \mathrm{kg}$ & $145 \pm 25$ & $149 \pm 44$ & $132 \pm 35$ & $143 \pm 37$ & $153 \pm 50$ & $166 \pm 58$ & $193 \pm 84$ \\
\hline Resting LDF (U) & $1 \mathrm{mg} / \mathrm{kg}+\mathrm{NE}$ & $153 \pm 57$ & $140 \pm 38$ & $187 \pm 63$ & $220 \pm 96$ & $243 \pm 90^{*}$ & $248 \pm 92$ & $258 \pm 82^{*}$ \\
\hline Resting LDF (U) & $5 \mathrm{mg} / \mathrm{kg}+\mathrm{NE}$ & $162 \pm 41$ & $174 \pm 57$ & $170 \pm 51$ & $208 \pm 58$ & $231 \pm 55$ & $247 \pm 79$ & $267 \pm 81^{*}$ \\
\hline Resting LDF (U) & $1 \mathrm{mg} / \mathrm{kg}+1400 \mathrm{~W}$ & $171 \pm 45$ & $156 \pm 47$ & $170 \pm 90$ & $176 \pm 53$ & $185 \pm 61$ & $195 \pm 63$ & $190 \pm 85$ \\
\hline Resting LDF (U) & $5 \mathrm{mg} / \mathrm{kg}+1400 \mathrm{~W}$ & $141 \pm 48$ & $120 \pm 40^{*}$ & $128 \pm 39$ & $140 \pm 58$ & $140 \pm 47$ & $141 \pm 41$ & $157 \pm 47$ \\
\hline
\end{tabular}

Data are given as mean \pm standard deviation. Statistical results to baseline are given as: ${ }^{\star} P<0.05,{ }^{\star \star} P<0.01,{ }^{\star \star \star} P<0.001$. BP $=$ blood pressure; EFVR = evoked flow velocity responses; LDF = laser-Doppler flowmetry; SEP = somatosensory evoked potentials. 
Table 3

Data from cytokine and destruction marker measurements as group averaged data \pm standard deviation

\begin{tabular}{llllll}
\hline & NSE ng/l & S-100B $\mathbf{~ g} / \mathrm{ml}$ & IL $\mathbf{6 ~ p g} / \mathbf{m l}$ & TNF- $\alpha$ pg/ml & IFN- $\gamma$ pg/ml \\
\hline Control & $0.29 \pm 0.14$ & $0.63 \pm 0.3$ & $93 \pm 28$ & $60 \pm 22$ & $32 \pm 3$ \\
$\mathbf{5} \mathbf{~ m g / k g ~ L P S ~}$ & $1.8 \pm 0.9$ & $13 \pm 8.6$ & $5498 \pm 1980$ & $1868 \pm 977$ & $1600 \pm 540$ \\
$\mathbf{5} \mathbf{~ m g / k g ~ L P S + 1 4 0 0 W}$ & $1.6 \pm 0.9$ & $11 \pm 9.7$ & $4998 \pm 1780$ & $1655 \pm 877$ & $1800 \pm 820$ \\
$\mathbf{5} \mathbf{~ m g / k g ~ L P S ~ + N E ~}$ & $2.2 \pm 0.4$ & $10 \pm 7.6$ & $5300 \pm 1654$ & $1285 \pm 592$ & $1960 \pm 660$ \\
\hline
\end{tabular}

Compared with control destruction markers and cytokine levels significantly increased in the sepsis groups but did not differ between sepsis groups.

1400W = N-(3-(aminomethyl)benzyl)acetamidine; IFN = interferon; IL = interleukin; LPS = lipopolysaccharide; NE = norepinephrine; NSE = neuron specific enolase.

$\mathrm{mmHg}$; both $P<0001$ to non-treated groups). Not as effective as NE $1400 \mathrm{~W}$ also stabilized blood pressure levels in both groups ( $82 \pm 12$ and $76 \pm 12 \mathrm{mmHg}$; both $P<0.001$ vs. nontreated animals).

Noteworthy is that only in the $1400 \mathrm{~W}$ groups glucose substitutions were necessary to maintain adequate blood glucose levels. NE led to even higher glucose levels as compared with the $1 \mathrm{mg} / \mathrm{kg}$ or $5 \mathrm{mg} / \mathrm{kg}$ LPS groups.

\section{Neurofunctional findings}

Addressing neurofunctional parameters NE was shown to be most effective on N2-P1 amplitudes. Compared with the 1 $\mathrm{mg} / \mathrm{kg}$ LPS group, evoked potential amplitudes were significantly higher throughout experiments (270 minutes: $16 \pm 3 \mu \mathrm{V}$ vs. $12 \pm 4 \mu \mathrm{V} ; P<0.05$; Figure 1$)$. In the $5 \mathrm{mg} / \mathrm{kg}$ group, NE prevented a progressive decline of amplitudes at the end of experiments ( 270 minutes: $14 \pm 3 \mu \mathrm{V}$ vs. $7 \pm 2 \mu \mathrm{V} ; P<0.001$; Figure 2). No protective effects were seen on the $\mathrm{P} 1$-latencies or evoked flow velocity responses (Table 2).

1400W led to an early and progressive decline in evoked potential amplitudes, which exceeded changes seen in the 1 $\mathrm{mg} / \mathrm{kg}$ or $5 \mathrm{mg} / \mathrm{kg}$ LPS groups (Figures 1 and 2). Similarly, P1latencies increased to a higher extent as expected from nontreated LPS groups (Table 2). Evoked flow velocity responses dropped in relation to the decrease in evoked potential amplitudes indicating still intact coupling. This makes the possibility of an artifact in electrical recordings unlikely.

\section{Discussion}

The functionally coupled blood flow responses are decreased during early phases of sepsis, which could contribute to brain dysfunction (sepsis-associated delirium, septic encephalopathy) in sepsis. Neither $1400 \mathrm{~W}$ nor NE improved the neurovascular coupling. However, interpretation of the effects of the iNOS-inhibition on the neurovascular coupling is hampered by the direct adverse effects of $1400 \mathrm{~W}$ on SEP, which were only seen under septic conditions. In both LPS groups, potential amplitudes declined and latencies increased directly after administration of the iNOS-inhibitor. In non-septic rats neither $1400 \mathrm{~W}$ nor other unspecific NO-inhibitors showed this effect $[24,25]$. A second new finding was the strong glucose lowering effect of $1400 \mathrm{~W}$ under septic conditions. From the literature a beneficial effect was anticipated because increased NO levels adversely interfere with the mitochondrial function and the intracellular glucose homeostasis [26,27]. However, occurrence of mitochondrial respiratory chain enzyme dysfunction was shown to occur at later stages beginning six to eight hours after sepsis induction $[28,29]$. Due to a tight glu-

Figure 1

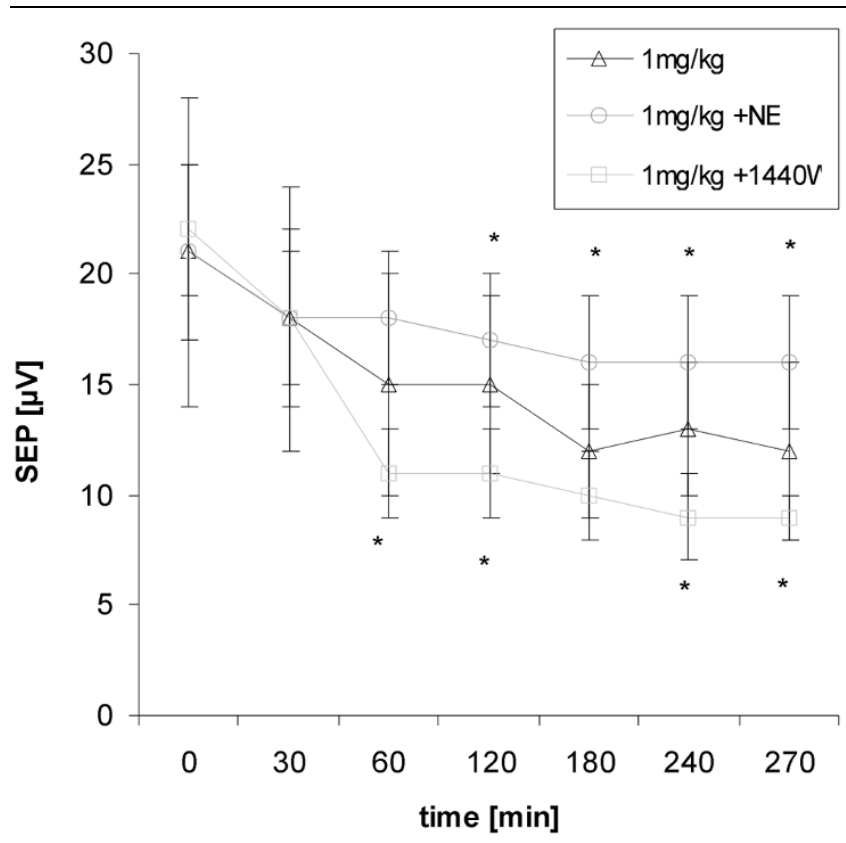

Time course of group averaged N2-P1 amplitudes given as mean \pm standard deviation for the $1 \mathrm{mg} / \mathrm{kg}$ lipopolysaccharide groups. Norepinephrine (NE) was protective on the potential amplitudes whereas selective inducible nitric oxide synthase (iNOS)-inhibition (N-(3-(aminomethyl)benzyl)acetamidine (1400W)) showed adverse effects. Statistical results are given as compared to the non-treated group; * $P<$ 0.05. SEP $=$ somatosensory evoked potentials. 
Figure 2

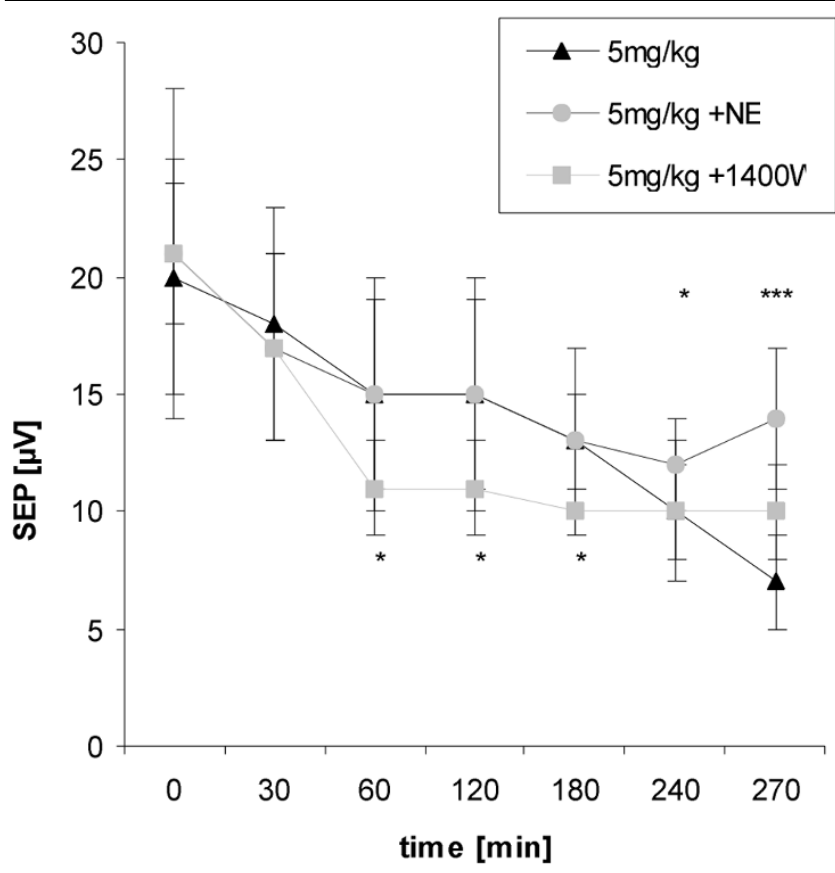

Time course of group averaged N2-P1 amplitudes given as mean \pm standard deviation for the $5 \mathrm{mg} / \mathrm{kg}$ lipopolysaccharide groups. Norepinephrine (NE) was protective on the potential amplitudes at the end of experiments whereas selective inducible nitric oxide synthase (iNOS)-inhibition (N-(3-(aminomethyl)benzyl)acetamidine (1400W)) showed again adverse effects from beginning of therapy. Sttistical results are given as compared with the non-treated group; * $P<0.05$; ${ }^{\star \star \star} P<0.001$.

cose control in the present study simple hypoglycemia cannot explain our findings.

The effect of NE on evoked potential amplitudes cannot be taken as a clear indication for neuroprotection. An improvement of amplitudes is a direct effect of NE, which has been described even under non-septic conditions [30]. It was explained by a more focused activation of cortical neuronal fields. In line with this interpretation, the oxidative metabolism of the brain did not change in septic patients under NE treatment [31]. The lack of an effect on the cell destruction markers also points against a significant neuroprotective effect. Regarding the cerebral circulation, NE is neutral as long as the blood-brain barrier is intact and exerts vasoconstrictive effects in case of a barrier leakage [32,33]. Therefore, the induced cerebral blood flow in the NE group could be best explained by the higher blood pressure levels. We also did not find an effect of NE on the neurovascular coupling. This is shown in Figure 3 which illustrates the relation between evoked potential amplitudes ( $x$-axis) and resultant flow velocity changes $(y-$ axis) from the beginning to end of experiments. Arrow 1 shows the typical initial uncoupling with a drop in evoked flow velocity responses but still intact evoked potential amplitudes. This response was not modified by NE as compared with non-
Figure 3

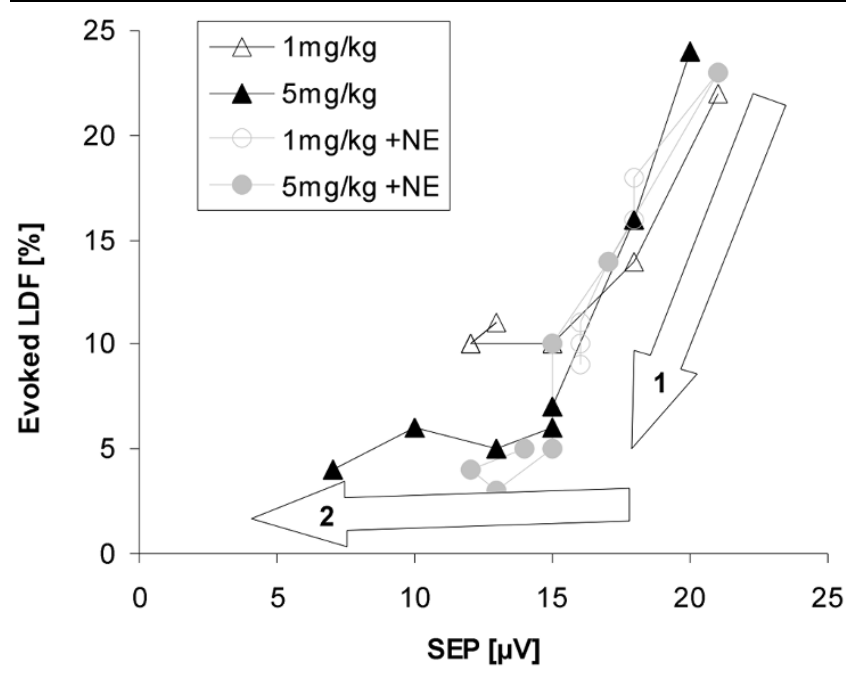

Graph of group averaged evoked potential amplitudes and evoked flow velocity responses to illustrate the temporal aspects of neurovascular dysfunction. With lipopolysaccharide (LPS) application it comes first to a disproportional high decline in evoked laser-Doppler responses in both LPS dose groups (arrow 1 ) before somatosensory evoked potential amplitudes declined (arrow 2). This constellation indicates early microcirculatory failure in the septic brain. Whereas norepinephrine (NE) did not modify the drop in hemodynamic responses (arrow 1 ) it was protective on the evoked potential amplitudes (absent $(1 \mathrm{mg} / \mathrm{kg}$ ) or diminished (5 mg/kg) component of arrow 2). LDF = laser-Doppler flowmetry; SEP = somatosensory evoked potentials.

treated groups. Arrow 2 shows the succeeding drop in evoked potential amplitudes, which were prevented in the NE group possibly due to a substance effect. The typical pattern of an initial uncoupling and succeeding drop in potentials was more pronounced in the $5 \mathrm{mg} / \mathrm{kg}$ groups as compared with the 1 $\mathrm{mg} / \mathrm{kg}$ groups.

Chloralose is a narcotic agent which allows neurophysiologic monitoring [34]. It results in a mild alkalosis which explains the higher initial pH levels.

We chose a classic catecholamine therapy, although immunomodulatory effects of some catecholamines were reported in the literature $[35,36]$. We did not find a significant effect of NE on the cytokine level and also did not find significant effects on the gene expression levels of chemokines [37]. Our data are therefore in line with others who found epinephrine but not NE to modulate cytokine levels in a porcine model of endotoxic shock [38].

The cell destruction markers NSE and S-100 calcium binding protein $B$ have been widely used to assess the prognosis and outcome of different disease processes [39-41]. A limitation occurs in case of a blood-brain barrier breakdown because serum levels then can considerably vary between individuals. A similar study excluded a blood-brain barrier breakdown for 
the first hours of a sepsis syndrome [33]. This is reflected by the narrow standard deviation of cell destruction markers.

\section{Conclusions}

Neurovascular coupling is decreased during early phases of sepsis. This could contribute to brain dysfunction in sepsis (sepsis-associated delirium). Neither NE nor 1400W considerably prevented the breakdown of the neurovascular coupling. However, further research is needed to clarify the direct adverse effects of $1400 \mathrm{~W}$ on neuronal function, which occurs only under septic conditions.

Key messages
Microcirculatory dysfunction occurs early in the septic
brain.
Besides its effects on blood pressure, norepinephrine
does not prevent the occurrence of sepsis-related cere-
bral microcirculatory failure and the effect on evoked
potential amplitudes seems to be a side effect of the
agent.
Under septic conditions, 1400 w stabilizes the blood
pressure but shows a direct adverse effect on evoked
potential amplitudes which does not appear under
physiologic conditions. Due to this effect, interpretation
of its effects on the neurovascular coupling is limited;
however, a clear beneficial effect was lacking.

\section{Competing interests}

The authors declare that they have no competing interests.

\section{Authors' contributions}

BR drafted the manuscript, performed the experiments together with SK and SW. SW additionally investigated cytokines and cell destruction markers. RS designed the experiments with BR and helped with writing the paper. All authors read and approved the final manuscript.

\section{Acknowledgements}

This project was funded by a Research Grant of the University Medical Centre Giessen and Marburg.

\section{References}

1. Hotchkiss RS, Karl IE: The pathophysiology and treatment of sepsis. New Engl J Med 2003, 348:138-150.

2. Parillo JE: Pathogenetic mechanisms of septic shock. New Engl J Med 1993, 328:1471-1478.

3. Tureen J: Effect of recombinant human tumor necrosis factoralpha on cerebral oxygen uptake, cerebrospinal fluid lactate, and cerebral blood flow in the rabbit: role of nitric oxide. J Clin Invest 1995, 95:1086-1091.

4. Vincent JL: Microvascular endothelial dysfunction: a renewed appreciation of sepsis pathophysiology. Crit Care 2001, 5:S1-S5.

5. Rees DD: Role of nitric oxide in the vascular dysfunction of septic shock. Biochem Soc Trans 1995, 23:1025-1029.

6. Rosselet A, Feihl F, Markert M, Gnaegi A, Perret C, Liaudet L: Selective iNOS inhibition is superior to norepinephrine in the treatment of rat endotoxic shock. Am J Respir Crit Care Med 1998, 157:162-170.
7. Scott JA, Mehta S, Duggan M, Bihari A, McCormack DG: Functional inhibition of constitutive nitric oxide synthase in a rat model of sepsis. Am J Respir Crit Care Med 2002, 165:1426-1432.

8. Gray G, Schott C, Julou-Schaeffer G, Fleming I, Parratt JR, Stoclet JC: The effect of inhibitors of the L-arginine/nitric oxide pathway on endotoxin-induced loss of vascular responsiveness in anaesthetized rats. Br J Pharmacol 1991, 103:1218-1244.

9. Hollenberg SM, Broussard M, Osman J, Parrillo JE: Increased microvascular reactivity and improved mortality in septic mice lacking inducible nitric oxide synthase. Circ Res 2000, 86:774-779.

10. MacMicking JD, Nathan C, Hom G, Chartrain N, Fletcher DS, Trumbauer M, Stevens K, Xie OW, Sokol K, Hutchinson N, Chen $\mathrm{H}$, Mudget JS: Altered responses to bacterial infection and endotoxic shock in mice lacking inducible nitric oxide synthase. Cell 1995, 81:641-650.

11. Wei XQ, Charles IG, Smith A, Ure J, Feng GJ, Huang FP, Xu D, Muller W, Moncada S, Liew FY: Altered immune responses in mice lacking inducible nitric oxide synthase. Nature 1995, 375:408-411.

12. Andresen J, Shafi NI, Bryan RM: Endothelial influences on cerebrovascular tone. J Appl Physio/ 2006, 100:318-327.

13. ladecola C: Neurovascular regulation in the normal brain and in Alzheimer's disease. Nat Rev Neurosci 2004, 5:347-360.

14. Buerk DG, Ances BM, Greenberg JH, Detre JA: Temporal dynamics of brain tissue nitric oxide during functional forepaw stimulation in rats. Neuroimage 2003, 18:1-9.

15. Okamoto $\mathrm{H}$, Ito $\mathrm{O}$, Roman RJ, Hudetz AG: Role of inducible nitric oxide snythase and cyclooxygenase-2 in endotoxin.induced cerebral hyperemia. Stroke 1998, 29:1209-1218.

16. Rosengarten $B$, Hecht $M$, Auch D, Ghofrani HA, Schermuly RT, Grimminger F, Kaps M: Microcirculatory dysfunction in the brain precedes changes in evoked potentials in endotoxin-induced sepsis syndrome in rats. Cerebrovasc Dis 2007, 23:140-147.

17. Ebersoldt M, Sharshar T, Annane D: Sepsis-associated delirium. Intensive Care Med 2007, 33:941-950.

18. Green R, Scott LK, Minagar A, Conrad S: Sepsis associated encephalopathy (SAE): a review. Front Biosci 2004, 9:1637-1641.

19. Pullamsetti SS, Maring D, Ghofrani HA, Mayer K, Weissmann N, Rosengarten B, Lehner M, Schudt C, Boer R, Grimminger F, Seeger W, Schermuly RT: Effect of nitric oxide synthase (NOS) inhibition on macro- and microcirculation in a model of rat endotoxic shock. Thromb Haemost 2006, 95:720-727.

20. Dirnagl U, Kaplan B, Jacewicz M, Pulsinelli W: Continuous measurement of cerebral cortical blood flow by laser-Doppler flowmetry in a rat stroke model. J Cereb Blood Flow Metab 1989, 9:589-596.

21. Ances BM, Detre JA, Takahashi K, Greenberg JH: Transcranial laser Doppler mapping of activation flow coupling of the rat somatosensory cortex. Neurosci Lett 1998, 257:25-28.

22. Lindauer $U$, Villringer $A$, Dirnagl $U$ : Characterization of $C B F$ response to somatosensory stimulation: model and influence of anesthetics. Am J Physiol 1993, 264:H1223-H1228.

23. Rosengarten $B$, Lutz $H$, Hossmann KA: A control system approach for evaluating somatosensory activation by laserDoppler flowmetry in the rat cortex. J Neurosci Methods 2003, 130:75-81.

24. Lindauer U, Megow D, Schultze J, Weber JR, Dirnagl U: Nitric oxide synthase inhibition does not affect somatosensory evoked potentials in the rat. Neurosci Lett 1996, 216:207-210.

25. Garvey EP, Oplinger JA, Furfine ES, Kiff RJ, Laszlo F, Whittle BJ Knowles RG: $1400 \mathrm{~W}$ is a slow, tight binding, and highly selective inhibitor of inducible Nitric-oxide synthase in vitro and in vivo. J Biol Chem 1997, 272:4959-4963.

26. Jobgen WS, Fried SK, Fu WJ, Meininger CJ, Wu G: Regulatory role for the arginine-nitric oxide pathway in metabolism of energy substrates. J Nutr Biochem 2006, 17:571-588.

27. Peres-Asensio FJ, Hurtado O, Burguete MC, Moro MA, Salom JB, Lizasoain I, Torregrosa G, Leza JC, Alborch E, Castillo J, Knowles RG, Lorenzo P: Inhibition of iNOS activity by $1400 \mathrm{~W}$ decreases glutamate release and ameliorates stroke outcome after experimental ischemia. Neurobiol Dis 2005, 18:375-384

28. Comim CM, Rezin GT, Scaini G, Di-Pietro PB, Cardoso MR, Petronilho FC, Ritter C, Streck EL, Quevedo J, Dal-Pizzol F: Mitochondrial respiratory chain and creatine kinase activities in rat brain 
after sepsis induced by cecal ligation and perforation. Mitochondrion 2008, 8:313-318.

29. d'Avila JC, Santiago AP, Amancio RT, Galina A, Oliveira MF, Bozza FA: Sepsis induces brain mitochondrial dysfunction. Crit Care Med 2008, 36:1925-1932.

30. Drouin C, Wang D, Waterhouse BD: Neurophysiological actions of methylphenidate in the primary somatosensory cortex. Synapse 2007, 61:985-990.

31. Moller K, Qvist T, Tofteng F, Sahl C, Sonderkaer S, Dethloff T, Knudsen GM, Larsen FS: Cerebral blood flow and metabolism during infusion of norepinephrine and propofol in patients with bacterial meningitis. Stroke 2004, 35:1333-1339.

32. Corday E, Williams JH Jr: Effect of shock and of vasopressor drugs on the regional circulation of the brain, heart, kidney and liver. Am J Med 1960, 29:228-241.

33. Rosengarten B, Walberer M, Allendoerfer J, Mueller C, Schwarz N, Bachmann G, Gerriets T: LPS-induced endotoxic shock does not cause early brain edema formation - A MRI study in rats. Inflamm Res 2008, 57:479-483.

34. Ueki M, Mies G, Hossmann KA: Effect of alpha-chloralose, halothane, pentobarbital and nitrous oxide anesthesia on metabolic coupling in somatosensory cortex of rat. Acta Anaesthesiol Scand 1992, 36:318-322.

35. Feinstein DL, Heneka MT, Gavrilyuk V, Gavrilyuk V, Dello Russo C, Weinberg G, Galea E: Noradrenergic regulation of inflammatory gene expression in brain. Neurochem Int 2002, 41:357-365.

36. Oberbeck R: Catecholamines: physiological immunomodulators during health and illness. Curr Med Chem 2006, 13:1979-1989.

37. Wolff S, Klatt S, Wolff JC, Wilhelm J, Fink L, Kaps M, Rosengarten $B$ : Endotoxin-induced gene expression differences in the brain and effects of iNOS inhibition and norepinephrine. Intensive Care Med 2009, 35:730-739.

38. Bergmann M, Gornikiewicz A, Tamandl D, Exner R, Roth E, Függer $R$, Götzinger $P$, Sautner T: Continuous therapeutic epinephrine but not norepinephrine prolongs splanchnic IL-6 production in porcine endotoxic shock. Shock 2003, 20:575-581.

39. Madl C, Grimm G, Kramer L, Yeganehfar W, Sterz F, Schneider B, Kranz A, Schneeweiss B, Lenz K: Early prediction of individual outcome after cardiopulmonary resuscitation. Lancet 1993, 341:855-858.

40. Nguyen DN, Spapen H, Su F, Schiettecatte J, Shi L, HachimiIdrissi $S$, Huyghens L: Elevated serum levels of $S-100 \beta$ protein and neuron-specific enolase are associated with brain injury in patients with severe sepsis and septic shock. Crit Care Med 2006, 34:1967-1974.

41. Wunderlich MT, Wallesch CW, Goertler M: Release of neurobiochemical markers of brain damage is related to the neurovascular status on admission and the site of arterial occlusion in acute ischemic stroke. J Neurol Sci 2004, 227:49-53. 